\title{
Ledge-directed epitaxy of continuously self-aligned single-crystalline nanoribbons of transition metal dichalcogenides
}

Areej Aljarb, 1,2 Jui-Han Fu,1 Chih-Chan Hsu,1,3 Chih-Piao Chuu,4 Yi Wan,1 Mariam Hakami, 1 Dipti R Naphade, 1 Emre Yengel, 1 Chien-Ju Lee,5 Steven Brems, 6 Tse-An Chen,4 Ming-Yang Li,4 Sang-Hoon Bae,7 Wei-Ting Hsu,8 Zhen Cao1, Rehab Albaridy,1 Sergei Lopatin,9 Wen-Hao Chang,5,8 Thomas D. Anthopoulos, 1 Jeehwan Kim, 7 LainJong $\mathrm{Li}_{1,1,4,10^{*}}$ and Vincent Tung ${ }^{*}$

1. Physical Sciences and Engineering Division, King Abdullah University of Science and Technology (KAUST), KAUST Solar Centre, Thuwal 23955-6900, Kingdom of Saudi Arabia

2. Department of Physics, King Abdulaziz University, Jeddah 21589, Kingdom of Saudi Arabia

3. Department of Electronics Engineering, National Tsing Hua University, Hsinchu 30013, Taiwan

4. Corporate Research, Taiwan Semiconductor Manufacturing Company (TSMC), 168

Park Ave. 2, Hsinchu Science Park, Hsinchu 30075, Taiwan

5. Department of Electrophysics, National Chiao Tung University, Hsinchu 30010 , Taiwan

6. Imec vzw, Kapeldreef 75, BE-3001 Leuven, Belgium

7. Department of Mechanical Engineering, Massachusetts Institute of Technology, Cambridge, MA 02139 USA

8. Center for Emergent Functional Matter Science (CEFMS), National Chiao Tung University, Hsinchu 30010, Taiwan

9. King Abdullah University of Science and Technology (KAUST), Core Labs, Thuwal, 23955-6900, Saudi Arabia

10. Department of Electronic Engineering, and Green Technology Research Center, Chang-Gung University, Taoyuan 333, Taiwan

*To whom correspondence should be addressed: ljliv@tsmc.com or vincent.tung@kaust.edu.sa

Two-dimensional (2D) transition metal dichalcogenides (TMDs) nanoribbons are touted as the future extreme device downscaling for advanced logic and memory devices but remain a formidable synthetic challenge. Here, we demonstrate a ledge-directed epitaxy (LDE) of dense arrays of continuous, selfaligned, monolayer and single-crystalline $\mathrm{MoS}_{2}$ nanoribbons on $\beta$-gallium (III) oxide $\left(\beta-\mathrm{Ga}_{2} \mathrm{O}_{3}\right)(100)$ substrates. LDE $\mathrm{MoS}_{2}$ nanoribbons have spatial uniformity over a long-range and transport characteristics on par with those seen in exfoliated benchmarks. Prototype MoS2 nanoribbon-based field-effect transistors exhibit high on/off ratios of 108 and an averaged room temperature electron 
mobility of $65 \mathrm{~cm}_{2} \mathrm{~V}_{-1 \mathrm{~S}}-1$. The $\mathrm{MoS}_{2}$ nanoribbons can be readily transferred to arbitrary substrates while the underlying $\beta-\mathrm{Ga}_{2} \mathrm{O}_{3}$ can be re-used after mechanical exfoliation. We further demonstrate LDE as a versatile epitaxy platform for the growth of p-type WSez nanoribbons and lateral heterostructures made of $p$-WSe2 and $n-M_{2} S_{2}$ nanoribbons for futuristic electronics applications.

Planar transistors have been used for myriad generations with size and voltage scaling to enhance performance and save cost, following the well-known Moore's Law1. Innovation of Fin-field effect transistor (Fin-FET) architecture was the solution and rendered the further device scaling possible. Unfortunately, short channel effect shall ultimately limit the Fin-FET scaling. A wave of revolutionary design in FET architecture with a superior gate control over the channel begins to take hold. This emerging stacked sheet architecture typically consists of multi-stacked semiconducting nanosheets with surrounding gate metals, which demonstrates better short-channel control and thus holds promise to extend the Moore's Law2. Aligned arrays of single-crystal, monolayer 2D TMDs nanoribbons with high aspect-ratios, which represent the ultimate limit of miniaturization in the vertical dimension, are therefore very attractive in this context. Specifically, the ability to achieve single crystallinity and electrical uniformity throughout the entirety of the 2D TMDs nanoribbons, the key metrics of enabling batch production FET arrays, would allow a very high degree of electrostatic control at very low-power consumption. Synthetic strategies, toward TMDs nanoribbons have been reported to individually achieve control of layer number, single-crystallinity, self-alignment, and dimensionalities3-5. However, the dearth of a manufacturing route toward TMDs nanoribbons that synergistically combine all the aforementioned properties remains a major challenge.

It is known that the lattice orientation of 2D TMDs can be guided by substrates through lifting the energy degeneracy of the 2D TMD-substrate van der Waals (vdW) system6. We have further revealed that the lateral docking of 2D hexagonal boron nitride $(h \mathrm{BN})$ seeds to the atomic step edges of $\mathrm{Cu}(111)$ substrates predominates over the vertical vdW registry of $h \mathrm{BN}$ on $\mathrm{Cu}$, ensuring the mono-orientated nucleation and thus achieving the growth of single-crystal $2 \mathrm{D} h \mathrm{BN}$ film7. These neat demonstrations of 
synthesizing the uniform monolayer 2D TMD films with single crystallinity highlight that the selection of substrate (e.g., thermodynamics) and the growth parameter control (e.g., kinetics) are critically important. Here, we explore the epitaxial growth of single crystalline and aligned TMDs nanoribbons via LDE assisted chemical vapor deposition (CVD), that hinges on the thermodynamic control of TMD seeding orientation in conjunction with the kinetic control of growth direction. Figure 1a illustrates the LDE growth scheme for $\mathrm{MoS}_{2}$ nanoribbons: (I) A single-crystal $\beta-\mathrm{Ga}_{2} \mathrm{O}_{3}$ (100) substrate with exposed ledges is used; (II) Nucleation of $\mathrm{MoS}_{2}$ seeds with preferred orientation takes place at the ledges of $\beta-\mathrm{Ga}_{2} \mathrm{O}_{3}$; (III) Aligned $\mathrm{MoS}_{2}$ domains merge into continuous nanoribbons; (IV) MoS2 nanoribbons can be easily peeled off from the $\beta-\mathrm{Ga}_{2} \mathrm{O}_{3}$ (100) substrate and readily transferred to arbitrary substrates via a polydimethylsiloxane (PDMS)-assisted process; and (V) Exfoliated $\beta-\mathrm{Ga}_{2} \mathrm{O}_{3}$ substrate can be re-used for another round of growth.

Intrinsically, the (100) plane of the freshly exfoliated $\beta-\mathrm{Ga}_{2} \mathrm{O}_{3}$ substrate exhibits atomically sharp steps with a step height of $\sim 6 \AA$ (half unit cell). These steps trend up and down across the entire $\beta-\mathrm{Ga}_{2} \mathrm{O}_{3}$ substrate, resulting in two sets of structurally equivalent but crystallographically inverted ledges namely (001) and (-201)8, 9 (Fig 1b). As featured in Figs. 1c-e, various stages in the growth of $\mathrm{MoS}_{2}$ nanoribbons are revealed by atomic force microscopy (AFM). Figure 1c clearly suggests that unidirectional nucleation of four $\mathrm{MoS}_{2}$ seeds carries out at the ledges. The edges of these triangular $\mathrm{MoS}_{2}$ seeds stay parallel to the well-defined step edge whereas the vertices point toward the lower terrace. Meanwhile, we observed that the nucleation density of oriented $\mathrm{MoS}_{2}$ seeds along both (001) and (-201) ledges is overwhelmingly higher than that of flat terraces where only sporadic distribution of randomly oriented MoS 2 flakes (orientation varies between $0^{\circ}, 90^{\circ}, 180^{\circ}$ and $270^{\circ}$ owing to the symmetry of $\beta-\mathrm{Ga}_{2} \mathrm{O}_{3}$ substrate, which is monoclinic in nature) can be spotted. The observation of unidirectional $\mathrm{MoS}_{2}$ seeding flakes on the atomically-textured, single crystalline $\beta-\mathrm{Ga}_{2} \mathrm{O}_{3}$ (100) substrate clearly indicates the existence of an energetically minimized MoS $2-\beta$ $\mathrm{Ga}_{2} \mathrm{O}_{3}$ ledge configuration, thus forming the basis for subsequent coalesce into continuous nanoribbons with single crystallinity. Indeed, aligned and mono-oriented $\mathrm{MoS}_{2}$ seeds grow by successive addition from the surrounding precursors and 
ultimately merge into a MoS2 nanoribbon as LDE approaches completion (Fig. 1e). The resulting $\mathrm{MoS}_{2}$ nanoribbons exhibit a uniform step height of $\sim 8 \AA$, characteristic of monolayer MoS2. Another unique capability of LDE is the controlled nucleation and unidirectional growth of ordered arrays of $\mathrm{MoS}_{2}$ nanoribbons at the atomic scale in previously unachievable quantities and scales (up to centimeter long and aspect ratios > 5,000 ). Images of AFM and scanning electron microscopy (SEM) collectively demonstrated the growth of dense arrays of globally aligned, continuous MoS2 nanoribbons enabled by LDE over the entire $\beta-\mathrm{Ga}_{2} \mathrm{O}_{3}(100)$ substrate (Figs. 1f-g).

In parallel, the innate step edges which are naturally present on the monolithic $\beta$ $\mathrm{Ga}_{2} \mathrm{O}_{3}(100)$ crystals10, have a propensity to cleave parallel to (100) and (001) planes by half unit cell. This is the result of unique octahedral arrangements of the Ga atoms (see Supplementary Fig.1) which are parallel to the (010) plane11. Consequently, the newly exfoliated (100) plane of $\beta-\mathrm{Ga}_{2} \mathrm{O}_{3}$ retains atomically clean, ordered and spatially distributed step edges with half-unit cell ledges as shown in Fig. $\mathbf{1}$ b. Photoluminescence $(\mathrm{PL})$ measurement taken on different batches of $\mathrm{MoS}_{2}$ nanoribbons grown on the repeatedly exfoliated $\beta-\mathrm{Ga}_{2} \mathrm{O}_{3}(100)$ substrate reveals neither changes in full width at half maximum (FWHM) nor the shift in PL peaks (see Supplementary Fig. 2), making it possible for the continuous and reliable batch production of high-quality MoS 2 nanoribbons. This peeling feature is particularly appealing as the ability to re-use $\beta-\mathrm{Ga}_{2} \mathrm{O}_{3}$ substrates eliminates the needs for a time-consuming and often laborious lithography process.

While all the aligned MoS2 flakes interlock in the same way and have the identical orientation, the atomic structures of $\beta-\mathrm{Ga}_{2} \mathrm{O}_{3}(100)$ has a profound implication on the geometric shapes of edges of MoS2 nanoribbons. Unlike the MoS2 flakes grown on a symmetrical substrate, $\mathrm{MoS}_{2}$ flakes grown on $\beta-\mathrm{Ga}_{2} \mathrm{O}_{3}(100)$ exhibit asymmetrically shaped edges, i.e., smooth and zigzag-shaped edges. Away from the well-defined ledges, the extremities of the merged MoS2 flakes are permitted to grow without any external constraint. The one edge of the single crystalline $\mathrm{MoS}_{2}$ nanoribbons assumes a regular zig-zag shape. High-angle annular dark-field scanning transmission electron microscopy (HAADF-STEM) Images in Supplementary Fig. 3 confirm the zigzag- 
shaped edges of the nanoribbons. Occasionally, we observed the formation of bilayer MoS2 nanoribbons. High-resolution (HR) HAADF-STEM images near the edges of the bilayer regions reveal the absence of Moiré patterns, indicating predominantly $2 \mathrm{H}$ stacking orders. Moreover, by controlling the growth temperature and nucleation density, the width of the MoS 2 nanoribbons can be systematically varied between $70 \mathrm{~nm}$ to $600 \mathrm{~nm}$ (see Supplementary Fig. 4), for which the width likely can meet the requirement for stacked sheet transistor applications. Further decrease in width for which fundamental confinement effects may arise, such as changes in bandgap and presence of 1D metallicity (Supplementary Discussion 1, and Fig. 5 and Fig. 6), is possible experimentally. Yet, the LDE TMD nanoribbons may not be continuous in the millimeter to centimeter length scale.

To verify the orientation of individual flakes and the associated crystallinity of the $M_{2} S_{2}$ nanoribbons, the LDE MoS2 nanoribbons were characterized by second harmonic generation (SHG) micro-spectroscopy and dark-field (DF) STEM. It is known that polarization-resolved SHG is sensitive to the crystal orientation and the intensity profile map can be used as a descriptor for verifying spatial orientations of the merged flakes within the coalesced nanoribbons12-14. Figure 2a displays the SHG intensity map taken from three horizontally aligned $\mathrm{MoS}_{2}$ nanoribbons with perpendicular polarization. All three $\mathrm{MoS}_{2}$ nanoribbons demonstrate homogenous SHG intensities except for a few nodes along the direction of laser irradiation. The discontinuity of SHG intensity is the result of rarely observed multilayer $\mathrm{MoS}_{2}$ seeds interspersed between the continuous $\mathrm{MoS}_{2}$ nanoribbons by comparison of AFM images (see Supplementary Fig. 3 and Fig. 7). The homogeneity of the SHG intensity proves that each nanoribbon indeed comprises $\mathrm{MoS}_{2}$ flakes with a single orientation. Furthermore, we deduced the angles between the laser polarization direction and the nearest armchair direction via the equation: $\theta=(1 / 3) \tan ^{-1} \sqrt{I_{x} / I_{y}} 12-14$. In this light, Fig. 2b features the intensity map that spatially resolves the angle distribution derived from compiling the simultaneously detected $I_{x}$ and $I_{y}$ SHG intensity, revealing uniform yet narrow angular distribution of 2o. The orientation of the zigzag direction is further confirmed by drawing comparisons of polarization resolved SHG intensity between the $\mathrm{MoS}_{2}$ nanoribbons and the reflected 
laser from the substrate. As indicated in the polar plot of Fig. 2c, MoS2 flakes with mirror domains of $0^{\circ}$ and $180^{\circ}$ orientations are angularly equivalent in terms of SHG intensity. The SHG can help to characterize the nanoribbons in a large area but the further distinguishing such mirror domains requires other methodologies.

It is known that the variation in crystallographic orientations disturbs the structural continuity, i.e., the formation of grain boundaries. This disruption manifests signs of polycrystalline in annular dark-field (ADF)-STEM on the nanometer length scale15 as shown in Fig. 2d (see Supplementary discussion 2). Mirror domains of $0^{\circ}$ (color in blue) and $180^{\circ}$ (color in yellowish gold) can therefore be clearly determined on the basis of convergent beam electron diffraction patterns (Fig. 2e). ADF-STEM images shown in Figs. 2f, $\mathbf{g}$ confirm the absence of mirror domains and thus the existence of crystallographic continuity of our LDE MoS2 nanoribbons on the micrometer length scale. This nanoribbon consists of more than twenty mono-oriented flakes and all ADFSTEM images exhibit crystallographically coherent domains with no visible grain boundaries, confirming the single-crystal nature of LDE MoS 2 nanoribbons. Other characterizations, including SEM image, corresponding PL mapping of the characteristic excitonic direct gap emission of monolayer $\mathrm{MoS}_{2}$, and signatures from Raman spectroscopy, again prove the structure continuity and crystallographic coherence of chemical states of MoS2 nanoribbons (Supplementary Fig. 8).

To understand the preferred nucleation at the ledge and the controlled growth along the step of $\beta-\mathrm{Ga}_{2} \mathrm{O}_{3}$, we conduct the cross-sectional HAADF HR-STEM (Figs. 3a, b) to provide the atomically resolved structures of both $\mathrm{MoS}_{2}$ nanoribbon and the underlying $\beta_{-\mathrm{Ga}_{2} \mathrm{O}_{3}}$ (100) substrate. Focus ion beam (FIB) was performed in the transverse direction of the $\mathrm{MoS}_{2}$ nanoribbon (perpendicular to the [010] of $\beta-\mathrm{Ga}_{2} \mathrm{O}_{3}$ ). The atomic structures of $\mathrm{MoS}_{2}$ nanoribbons are divided into three regions based on the locations: namely, (I) bottom terrace (left), (II) ledge (center), and (III) top terrace (right), respectively, allowing us to elucidate the relationship between epilayer and growth substrate. In agreement with the AFM image (Fig. 1d), region (II), the center segment of the nanoribbons where the nucleation of aligned, triangular seeds takes place, is found to lay above the $(-201)$ ledge of $\beta-\mathrm{Ga}_{2} \mathrm{O}_{3}$ (Fig. 3b). This preferred alignment of triangular 
seeds reveals that $(-201)$ ledge may represent the preferential nucleation site with the local energetic minimum. With this assumption, we first examine the effect of preferred nucleation sites along the (-201) edges through constructing a cross-sectional atomic model for $\beta-\mathrm{Ga}_{2} \mathrm{O}_{3}(100)$. Here, the $\beta-\mathrm{Ga}_{2} \mathrm{O}_{3}(100)$ substrate has a monoclinic structure with lattice constants of $\mathbf{a}=3.037 \AA, \mathbf{b}=5.798 \AA$, and $\beta=103.8^{\circ}{ }_{11}$. Two possible nucleation cases are proposed and their binding energies are calculated: (1) case $A$, where a $\mathrm{Ga}$ atom is notably missing from the vicinal (-201) ledge (Figs. 3b,c); and (2) case $B$, whereas $G$ a atoms remain intact near the (-201) ledge (Supplementary Fig. 9). In case $\mathrm{A}, \mathrm{MoS}_{2}$ molecules with $0^{\circ}$ and $180^{\circ}$ orientations are used as nuclei and are intentionally placed in the vicinity of $(-201)$ edges on $\beta-\mathrm{Ga}_{2} \mathrm{O}_{3}(100)$ as schematically represented in Figs. 3c,d. After relaxation, we find that $\mathrm{MoS}_{2}$ molecule with $0^{\circ}$ orientation predominately dock at the binding sites of (-201) edges.

Meanwhile, first-principles calculations revealed a drastic difference in binding energy of $\sim 2 \mathrm{eV}$ relative to that of inversely orientated MoS2 molecule $\left(180^{\circ}\right)$, thus favoring the mono-oriented growth and therefore the unidirectional alignment. An opposite trend is observed in case B (Supplementary Fig. 9), but there exhibits an energy difference of only $\sim 0.535 \mathrm{eV}$ when the $\mathrm{MoS}_{2}$ molecules dock to the oxygen at the bottom of $(-201)$ ledge. Unlike case $A$ where $0^{\circ}$ is the preferred orientation, the preferred orientation in case $B$ is $180^{\circ}$ which will lead to mirror grain boundaries in the ribbons (see Supplementary Discussion 3 and Fig. 10). Certainly, the mono-oriented seeds in our nanoribbons are nucleated following the favorable nucleation case (case A) due to the fact that $\mathrm{Ga}$ vacancies are naturally present near the edge of the steps (see Supplementary Fig. 11).

The proposed mechanism toward unidirectional nucleation is similar to the recently reported defect-enhanced degeneracy-breaking of TMDs16, but is quite independent due to the difference in spatial arrangement of docking sites-randomly distributed and disorganized defect sites vs. spatially ordered and aligned ledge sites. Nevertheless, in this work, they observed the reversal of triangle orientation (i.e., $0^{\circ}$ becomes $180^{\circ}$ ) of MoS2 flakes across a step edge in the $h \mathrm{BN}$ substrate under the assumption of a change in layer polarity of $\mathrm{AA}^{\prime}$-stacked $h \mathrm{BN}$. On the contrary, the two 
energetically equivalent but crystallographically inverted ledges, (-201) vs. (001) revealed by DF-STEM and atomic models, across the step edges of $\beta-\mathrm{Ga}_{2} \mathrm{O}_{3}(100)$, thus guiding the alignment of $\mathrm{MoS}_{2}$ nuclei in $0^{\circ}$ and $180^{\circ}$ orientations respectively (see Supplementary Discussion 3 and Fig. 11). Once the mono-oriented nucleation approaches completion, the rich sulfur (S) environment not only helps to break the vdW interaction between the aligned $\mathrm{MoS}_{2}$ seeds and the ledges, but also facilities the growth of single crystalline domains extended beyond both ends of the step edge, ultimately merging together into a continuous nanoribbon.

We note that growth of individual domains which strongly depends on the diffusion path, seems to be confined and directed along the ledges of $\beta-\mathrm{Ga}_{2} \mathrm{O}_{3}$ (100). This is very intriguing as the growth of TMDs on highly symmetric substrates by means of a CVD typically results in the omnidirectional diffusion of precursor vapors to the local environment. To verify the origin of this directional diffusion pathway, we performed a potential energy surface (PES) mapping of the (-201) plane of $\beta-\mathrm{Ga}_{2} \mathrm{O}_{3}(100)$ via density function theory (DFT) calculations. As shown in Fig. 3e, the surface diffusion kinetics along the [010] energetically confine the growth of $\mathrm{MoS}_{2}$, thus driving the energetically favorable and directionally modulated growth of aligned domains into single crystalline nanoribbons. These findings collectively point toward an entirely novel strategy to synthesize dense arrays of single crystalline, and globally aligned TMD monolayer nanoribbons for device applications.

The success of creating extended, single-crystal $\mathrm{MoS}_{2}$ nanoribbons is manifested in the uninterrupted, homogenous yet narrow distribution of signature $\mathrm{PL}$ wavelength across the aligned domains, indicating the lack of atomic misfits between merged domains as shown in Fig. 4a, and Supplementary Fig. 12a. Meanwhile, hyperspectral PL mapping, which provides a fast, global mapping with high spatial and spectral resolution, does not reveal any sign of PL quenching typically associated with grain boundaries. Results from conductive (C-) AFM on the MoS2 nanoribbons directly grown on a semiconducting $\beta-\mathrm{Ga}_{2} \mathrm{O}_{3}$ substrate show the similar trend in the representative topography and corresponding current maps are shown in Fig. $\mathbf{4 b}$. The local point current-voltage (I-Vs, vertical transport) and current mapping were done by 
applying a positive bias to $\beta-\mathrm{Ga}_{2} \mathrm{O}_{3}$ substrate while the conductive tip (Pt-Ir) was held at ground (Supplementary Figs. 12b,c). The MoS2 nanoribbons appear highly conducting relative to that of the underlying $\beta-\mathrm{Ga}_{2} \mathrm{O}_{3}$ substrate, making them clearly visible in the current map. The average current flowing throughout the $\mathrm{MoS}_{2}$ nanoribbons in the vertical direction is $18( \pm 2) \mathrm{nA}$. The point I-V curve measured along the MoS2 nanoribbons exhibits non-ohmic characteristics that appear symmetric. These measurements provide direct experimental evidence of the undisruptive conductive path throughout the entirety of MoS2 nanoribbons.

Furthermore, we verified the quality of $\mathrm{MoS}_{2}$ nanoribbons by evaluating the fieldeffect carrier mobility in a bottom-gate transistor configuration as illustrated in Fig. 4c and Supplementary 13a-d. To reduce the screening effect from the $\mathrm{HfO}_{2}$, while eliminating the charge scattering and trap sites, a single-crystal $h \mathrm{BN}$ monolayer film is embedded as an interface layer between $\mathrm{HfO}_{2}$ and $\mathrm{MoS}_{2}$ nanoribbons7. Noted that measurements were performed at room temperature. The electrical properties of our LDE MoS 2 nanoribbons have two important features: the spatial uniformity over a long range similar to those wafer-scale films synthesized by CVD/MOCVD and transport characteristics on par with those seen in exfoliated counterparts. Top panel of Supplementary $13 \mathrm{e}$ plots field-effect mobility and on/off ratios measured from five devices, fabricated on the same MoS 2 nanoribbon and separated by up to $20 \mu \mathrm{m}$ on a single chip. All five FETs exhibit nearly identical behaviors. These include an averaged field-effect mobility of $65 \mathrm{~cm} 2 / \mathrm{V}$-s and on/off ratios near $\sim 108$ independent of channel length and location of $\mathrm{MoS}_{2}$ nanoribbons, suggesting the spatial homogeneity of the electrical properties of the MoS2 nanoribbons across various length scales. Specifically, both values are comparable to the performance of the mechanically exfoliated benchmarks as shown in Fig. $\mathbf{4} \mathbf{d}_{17}$. In parallel, bottom panel of the Supplementary $\mathbf{1 3 e}$ provides the histogram of field-effect mobility and on/off ratios measured from 100 FETs made of different batches of $\mathrm{MoS}_{2}$ nanoribbons. Evidently, single crystallinity throughout the entirety of $\mathrm{MoS}_{2}$ nanoribbons is attested by the very narrow distributions of both field-effect mobility and on/off ratios. Occasionally, we find that field-effect mobility of $\mathrm{MoS}_{2}$ nanoribbons FETs exceeds $100 \mathrm{~cm}_{2} \mathrm{~V}_{-1} \mathrm{~S}-1$, with the highest value of $109 \mathrm{~cm}_{2} \mathrm{~V}_{-1} \mathrm{~S}-1$ 18. The mobility is enhanced due to the synergistic effect between $h \mathrm{BN}$ layer and $\mathrm{MoS}_{2}$ 
nanoribbons which are both single crystal in nature, ensuring the smooth charge transport along the heterointerface channel (Supplementary Fig. 14).

The location-selective hyperspectral PL included in Supplementary Fig. 15a-c revealed that both PL peak positions and FWHM did not vary significantly when the focus of laser spot was moved across the LDE MoS2 nanoribbon, characteristic of the uniform quality and continuous crystallinity of LDE MoS2 nanoribbons. In parallel, the low temperature PL (Supplementary Figs. 15d,e, excitation: $532 \mathrm{~nm}$, power: $200 \mu \mathrm{W}$ ) shows characteristics unique to exfoliated monolayer $\mathrm{MoS}_{2}$ benchmarks, including comparable PL intensity, a similar level of defects, neutral exciton and trion emission peaks, further confirming the high quality of LDE MoS2 nanoribbons. CVD grown-MoS2 typically exhibits a high density of defects even though these specimens are characterized as high crystallinity. As a consequence, PL induced from defects of CVDsynthesized TMD emerges and outweighs the intrinsic $\mathrm{PL}$ at $4 \mathrm{~K}$ unless treated chemically or doped electrostatically 19,20 . The result is the impaired transport property and decreased mobility21. This finding of containing a low-level defect density and preserving the high level of single crystallinity simultaneously during the growth stage has not been reported or achieved elsewhere and thus distinguishes the LDE from the other epitaxy approaches.

Because our LDE growth is directed by the combination of ledge sites and surface diffusion limited pathway, intrinsic to the $\mathrm{Ga}_{2} \mathrm{O}_{3}$ substrates, its use is not limited to the $\mathrm{MoS}_{2}-\mathrm{Ga}_{2} \mathrm{O}_{3}$ combination reported here. Instead, it could be generalized for producing various TMD nanoribbons, including $\mathrm{n}-\left(\mathrm{MoS}_{2}\right), \mathrm{p}-\left(\mathrm{WSe}_{2}\right)$ and even lateral $\mathrm{n}$ $\left(\mathrm{MoS}_{2}\right)-\mathrm{p}(\mathrm{WSe} 2)-\mathrm{n}\left(\mathrm{MoS}_{2}\right)$ junctions with precise single-crystallinity, alignment and monolayer controls over a micro-to-centimeter scale (Supplementary Fig. 16). While TMD nanoribbons with lateral heterostructures have been recently reported by a vaporliquid-solid growth4, such a process only allows the growth of heterostructures with either different metals or chalcogen atoms, thus making it challenging for the creation of $p-n$ heterostructures or even n-p-n multi-heterostructures. LDE WSe2-MoS2 lateral n-p-n multi-heterojunctions are achieved by growing WSe2 nanoribbons first on $\beta-\mathrm{Ga}_{2} \mathrm{O}_{3}$ (100) substrates, followed by the edge epitaxy of $\mathrm{MoS}_{2}$ nanoribbons on both sides as shown 
in Supplementary Fig. 17a. Hyperspectral PL mapping of relevant PL characteristics, including $\mathrm{MoS}_{2}$ in red, and WSe2 in blue, in tandem with Raman and PL spectra, evidentially proves the successful in-plane growth of $n$-type $\mathrm{MoS}_{2}$ at both edges of $p$ type WSe2 (Supplementary Fig. 17b-d).

\section{Acknowledgments:}

V.T., and J.-H.F. are indebted to the support from the King Abdullah University of Science and Technology (KAUST) Office of Sponsored Research (OSR) under Award No: OSR-2018-CARF/CCF-3079. V.T. acknowledges the support from KAUST Catalysis Center (KCC) and physical science division. C.P.C., T.A.C., M.Y.L., and L.J.L. thanks the support from Taiwan Semiconductor Manufacturing Company (TSMC). W.H.C. acknowledges the supports from the Ministry of Science and Technology of Taiwan (MOST-108-2119-M-009-011-MY3, MOST-107-2112-M-009-024-MY3) and from the CEFMS of NCTU supported by the Ministry of Education of Taiwan. V.T. and A.A. are grateful to Prof. Chen-Hsin Lien, and Prof. Luigi Cavallo for their support, Dr. HaoLing Tang, Dr. Ming-Hui Chiu, and Chien-Chih Tseng for the assistance of device architecture and chemical vapor deposition.

\section{Author statements:}

A.A., L.J.L. and V.T. conceived the project. A.A., J.-H.F., Y.W., M.H., and R.A. performed the synthesis of TMDs nanoribbons and heterostructures, and carried out Raman, PL, and AFM characterizations. C.C.H., T.A.C., M.-Y.L., and J.-H.F. conducted fabrication of field-effect transistors and associated calculations. D.R.N., E.Y. and T.A. performed and analyzed C-AFM and Hyper PL spectra. S.B. synthesized and provided the single-crystal $\mathrm{Cu}$ (111) for hBN. S.-H.B. and J.K. transferred $2 \mathrm{D}$ TMD and heterostructures. C.P.C. and Z.C. performed the first-principles calculations. A.A., S.L. and J.-H.F. accomplished the DF-STEM, and cross-sectional HAADF-STEM. C.-J.L., W.-T.H. and W.-H.C. executed the SHG analysis. All of the authors discussed and contributed to the results. A.A., L.J.L. and V.T. wrote the paper.

\section{Competing interests:}

The authors declare no competing interests. 


\section{Methods:}

Growth and Transfer of $\mathrm{MoS}_{2}$ and WSe2 nanoribbons. Single-crystal $\mathrm{MoS}_{2}$ and WSe2 monolayer nanoribbons were grown on the $\beta-\mathrm{Ga}_{2} \mathrm{O}_{3}(100)$ substrate by the conventional chemical vapor deposition (CVD) in a horizontal hot-wall 2" furnace tube with two heating zones. High purity of the S (Sigma-Aldrich, 99.99\%), Se (Sigma-Aldrich, 99.99\%), $\mathrm{MoO}_{3}$ (Sigma-Aldrich, 99.9\%) and $\mathrm{WO}_{3}$ (Sigma-Aldrich, 99.9\%) powders were used as the reaction precursors. $\mathrm{MoO}_{3}\left(\mathrm{WO}_{3}\right)$ powder typically was placed in a ceramic boat and was put in the heating zone centre of the furnace. S $(\mathrm{Se})$ powder was placed in a separate quartz boat at the upper stream side maintained at $140 \circ \mathrm{C}(270 \circ \mathrm{C})$ during the reaction. The single-crystal $\beta-\mathrm{Ga}_{2} \mathrm{O}_{3}(100)$ substrate was placed at the downstream side, where the precursor vapours were brought to the substrates by an argon ( $\mathrm{Ar})$ flowing gas at 30 torr for MoS2 and $\mathrm{Ar} /$ hydrogen $\left(\mathrm{H}_{2}\right)$ mixture gas at 10 torr for WSe2. The centre heating zone was heated to $800 \circ \mathrm{C}$ and kept for 10 min for the growth of $\mathrm{MoS}_{2}$ nanoribbons. On the other hand, for the growth of WSez nanoribbons, the furnace was heated to $900 \circ \mathrm{C}$ and was held for $15 \mathrm{~min}$. Upon completion of growth, the furnace was naturally cooled down to room temperature.

Transfer of Monolayer $\mathrm{MoS}_{2}$ nanoribbons. After the CVD growth, the resulting $\mathrm{MoS}_{2}$ nanoribbons on $\beta-\mathrm{Ga}_{2} \mathrm{O}_{3}(100)$ were transferred onto a substrate of interest via a polydimethylsiloxane (PDMS)-assisted approach. In brief, a thin PDMS film was placed on top of $\mathrm{MoS}_{2} / \beta-\mathrm{Ga}_{2} \mathrm{O}_{3}$. Note that it is very critical to ensure conformal contact between $\mathrm{PDMS}$ and $\mathrm{MoS}_{2} / \beta-\mathrm{Ga}_{2} \mathrm{O}_{3}$. Next, the PDMS/MoS $2 / \beta-\mathrm{Ga}_{2} \mathrm{O}_{3}$ stacked film was soaked in a $1 \mathrm{M} \mathrm{KOH}$ for $5 \mathrm{~min}$ at room temperature, followed by rinsing the sample with a copious amount of deionized (DI) water, PDMS/MoS2 stacked film was slowly peeled-off from $\beta$ $\mathrm{Ga}_{2} \mathrm{O}_{3}$ and then placed on a target substrate. The sample was kept in a vacuum for 30 min to make sure the adhesion between $\mathrm{MoS}_{2}$ and the target substrate. Residual water droplets were dried under a constant nitrogen $\left(\mathrm{N}_{2}\right)$ flow. Finally, PDMS was peeled off, leaving behind the MoS2 nanoribbons on the target substrate.

Characterizations. A FEI Quanta 600 scanning electron microscopy (SEM) was utilized to provide morphological views operating at $5 \mathrm{kV}$. Raman and photoluminescence $(\mathrm{PL})$ spectra on $\mathrm{MoS}_{2}, \mathrm{WSe} 2$ nanoribbons and the lateral heterostructures of both were 
collected using a Witec alpha 300 confocal Raman microscope equipped with a RayShield coupler. A 532-nm solid state laser as the excitation source. The excitation light with a power of $2.5 \mathrm{~mW}$ was focused onto the sample by a 100X objective lens (N.A. = 0.9). The signal was collected by the same objective lens, analyzed by a $0.75-\mathrm{m}$ monochromator and detected by a liquid-nitrogen-cooled CCD camera. The atomic force microscopy (AFM) characterizations were conducted with Olympus (OMCLAC240TS) Al-coated silicon cantilevers. The resonance frequency was $\sim 70 \mathrm{kHz}$, the spring constant was $\sim 2 \mathrm{~N} / \mathrm{m}$, and the tip curvature radius was $\sim 7 \mathrm{~nm}$. The C-AFM measurements were conducted using Pt-Ir-coated conductive probes (SCM-PIT, Bruker) with a spring constant in the range of 0.5 to $4 \mathrm{Nm}-1$. Contact mode was utilized for imaging. A constant tip to sample bias of $+3 \mathrm{~V}$ was applied during all the C-AFM scans. AFM raw images were processed using a Gwydion 2.51 software. The cross-sectional scanning transmission electron microscopy (STEM) samples were prepared in a Helios NanoLab 660 DualBeam focused ion beam (FIB) system. Cross-sectional HR-STEM imaging was conducted using a Thermofisher USA (former FEI) Titan Themis Z transmission electron microscope (TEM) equipped with a double Cs (spherical aberration) corrector operating at $300 \mathrm{kV}$. Dark field (DF)-STEM with High-angle annular dark-field (HAADF) imaging was done in a scanning mode using an acceleration voltage of $80 \mathrm{kV}$ with a column (at the sample) vacuum of about 2-4X10-7 Torr at room temperate. Hyper-spectral photoluminescence $(\mathrm{PL})$ measurements and mapping were taken with an IMA ${ }^{\mathrm{TM}}$ hyperspectral microscope from Photon Inc. The samples were excited from a $532 \mathrm{~nm}$ laser with the intensity of $6.4 \mu \mathrm{W} / \mu \mathrm{m} 2$ and spectra were collected from an area of $90 \mu \mathrm{m} \times 65 \mu \mathrm{m}$ with $1 \mathrm{~nm}$ resolution. Exposure time was chosen as 120 sec for each wavelength.

Second Harmonic Generation (SHG) measurements. A home-built microscope arranged in the backscattering set-up was utilized to measure the SHG signals. Laser pulses generated from a mode-locked titanium (Ti)-sapphire laser with a wavelength of $850 \mathrm{~nm}$, a pulse width of $\sim 150$ fs and a repetition rate of $80 \mathrm{MHz}$ were used as the fundamental laser field. The polarization was selected by a linear polarizer and a half waveplate. The laser beam which illuminates normally onto the sample was focused by a $100 \mathrm{X}$ objective lens with a N.A. of 0.9 . The generated SHG signals were collected by 
the same objective and sent to a $0.75 \mathrm{~m}$ spectrometer equipped with a liquid $\mathrm{N}_{2}$ cooled CCD camera. The scattered fundamental laser field was blocked by a $450 \mathrm{~nm}$ short pass filter. For the measurement of polarization resolved SHG images, the sample was mounted on a motorized $x-y$ stage and scanned with a step size of $0.2 \mu \mathrm{m}$. The SHG signals were sent through a polarizing beam-splitter to separate the SHG intensity with polarizations that are parallel and perpendicular to the laser polarization. The two components were sent into the spectrometer and detected by the CCD camera simultaneously. For the polarization resolved $\mathrm{SHG}$, the orientation of $\mathrm{MoS}_{2}$ nanoribbons was aligned closely to the polarization direction of the excitation laser. The linear polarization of the excitation laser and the SHG signals were selected and analyzed by the combination of a half waveplate and a linear polarizer. The polarization of the generated signals was rotated by a half waveplate mounted on a motorized rotational stage with a step of $4 \circ$ and analyzed by the polarizer.

First-principles Calculation. The first-principles calculations were carried out based on the density function theory (DFT) as implemented in the Vienna Ab-initio Simulation Package (VASP)22 within the MedeA23. The texchange-correlation potential described by the PBE-GGA24, and the van der Walls (vdW) correction vdW-DF (optB86b) functional 25 is used to calculate the binding energy difference between MoS2 molecules and $\beta-\mathrm{Ga}_{2} \mathrm{O}_{3}$ substrates. The $\beta-\mathrm{Ga}_{2} \mathrm{O}_{3}$ has a monoclinic structure with lattice constants of $a=3.037 \AA, b=5.798 \AA$, and $\beta=103.8^{\circ}{ }_{11}$. A k-grid of $8 \times 1 \times 1$ and an energy cut-off $400 \mathrm{eV}$ were used for the system of $5 \mathrm{MoS}_{2} / 1 \times 5 \beta-\mathrm{Ga}_{2} \mathrm{O}_{3}$, with a vacuum of $20 \AA$ along $\mathrm{c}$ and at least $10 \AA$ along $b$ between $\mathrm{MoS}_{2}$ molecule and the other side of ledge in a periodic cell to eliminate spurious interaction.

Field-Effect Transistor Fabrication and Measurement. Monolayer $\mathrm{MoS}_{2}$ nanoribbons grown on $\beta-\mathrm{Ga}_{2} \mathrm{O}_{3}$ (100) were transferred on $15 \mathrm{~nm} \mathrm{HfO}$, which was deposited on heavily doped silicon ( $\mathrm{Si}$ ) via the atomic layer deposition (ALD) as a gate insulator. A single crystalline $h \mathrm{BN}$ monolayer was detached from the $\mathrm{Cu}(111) /$ sapphire substrate by electrochemical delamination and then transferred onto $\mathrm{HfO}_{2} / \mathrm{Si}$ via a combination of thermal release tape (TRT; \#3195M) and poly (methyl methacrylate) (PMMA). The TRT can be released by annealing the TRT/PMMA/hBN/HfO$/ 2$ si stacked films on a hotplate 
at $180^{\circ} \mathrm{C}$. The PMMA film was thoroughly removed via iteratively immersing the sample in a hot acetone bath for $40 \mathrm{~min}$, leaving behind a $h \mathrm{BN} / \mathrm{HfO}_{2} / \mathrm{Si}$ stacked substrate. After transferring the $\mathrm{MoS}_{2}$ nanoribbons, the resulting $\mathrm{MoS}_{2}$ nanoribbons $/ h \mathrm{BN} / \mathrm{HfO}_{2} / \mathrm{Si}$ were placed in a vacuum chamber under a pressure of 10-6 torr for $12 \mathrm{~h}$. Owing to the global alignment of LDE grown MoS2 nanoribbons that provides far fewer constraints for the effective fabrication of field-effect transistors, electron-beam lithography emerges as the reliable method for producing the patterns of metal electrodes comprised of nickel $(\mathrm{Ni}$, $20 \mathrm{~nm}$ ) and gold ( $\mathrm{Au}, 50 \mathrm{~nm}$ ) necessary for electrical testing. More than one hundred single-nanoribbon field-effect transistors were reliably produced, and all tested to confirm the electrical output performance. This is due to the uniform, self-aligned, and tunable distribution of $\mathrm{MoS}_{2}$ nanoribbons over the entire area of the $\beta-\mathrm{Ga}_{2} \mathrm{O}_{3}$ (100) substrate $(\sim 1 \mathrm{~cm} \times 1.5 \mathrm{~cm})$. All measurements were carried out under ambient conditions using a Keithley 4200 semiconductor analyzer.

\section{Data availability:}

The data from this study are available from the corresponding author on reasonable request.

\section{References and Notes:}

1. Kang, K.; Xie, S.; Huang, L.; Han, Y.; Huang, P. Y.; Mak, K. F.; Kim, C.-J.; Muller, D.; Park, J., High-mobility three-atom-thick semiconducting films with wafer-scale homogeneity. Nature 520, 656-660 (2015).

2. Li, M.-Y.; Su, S.-K.; Wong, H.-S. P.; Li, L.-J., How 2D semiconductors could extend Moore's law. Nature (2019).

3. Hung, Y. H.; Lu, A. Y.; Chang, Y. H.; Huang, J. K.; Chang, J. K.; Li, L. J.; Su, C. Y., Scalable Patterning of MoS2 Nanoribbons by Micromolding in Capillaries. ACS Appl. Mater. Interfaces 8, 20993-1001 (2016).

4. Li, S.; Lin, Y.-C.; Zhao, W.; Wu, J.; Wang, Z.; Hu, Z.; Shen, Y.; Tang, D.-M.; Wang, J.; Zhang, Q., Vapour-liquid-solid growth of monolayer MoS 2 nanoribbons. Nat. Mater. 17, 535-542 (2018).

5. Chowdhury, T.; Kim, J.; Sadler, E. C.; Li, C.; Lee, S. W.; Jo, K.; Xu, W.; Gracias, D. H.; Drichko, N. V.; Jariwala, D.; Brintlinger, T. H.; Mueller, T.; Park, H.-G.; Kempa, T. J., Substrate-directed synthesis of MoS2 nanocrystals with tunable dimensionality and optical properties. Nat. Nanotechnol. 15, 29-34 (2020).

6. Kong, W.; Li, H.; Qiao, K.; Kim, Y.; Lee, K.; Nie, Y.; Lee, D.; Osadchy, T.; Molnar, R. J.; Gaskill, D. K.; Myers-Ward, R. L.; Daniels, K. M.; Zhang, Y.; Sundram, S.; Yu, Y.; Bae, S. H.; Rajan, S.; Shao-Horn, Y.; Cho, K.; Ougazzaden, A.; Grossman, J. C.; Kim, J., Polarity governs atomic interaction through two-dimensional materials. Nat. Mater. 17, 999-1004 (2018). 
7. Chen, T. A.; Chuu, C. P.; Tseng, C. C.; Wen, C. K.; Wong, H. P.; Pan, S.; Li, R.; Chao, T. A.; Chueh, W. C.; Zhang, Y.; Fu, Q.; Yakobson, B. I.; Chang, W. H.; Li, L. J., Waferscale single-crystal hexagonal boron nitride monolayers on $\mathrm{Cu}$ (111). Nature 579, 219-223 (2020).

8. Mu, W.; Jia, Z.; Yin, Y.; Hu, Q.; Zhang, J.; Feng, Q.; Hao, Y.; Tao, X., One-step exfoliation of ultra-smooth $\beta$-Ga2O3 wafers from bulk crystal for photodetectors. CrystEngComm 19, $5122-5127$ (2017).

9. $\quad$ Schewski, R.; Lion, K.; Fiedler, A.; Wouters, C.; Popp, A.; Levchenko, S. V.; Schulz, T.; Schmidbauer, M.; Bin Anooz, S.; Grüneberg, R.; Galazka, Z.; Wagner, G.; Irmscher, K.; Scheffler, M.; Draxl, C.; Albrecht, M., Step-flow growth in homoepitaxy of $\beta$-Ga2O3 (100)-The influence of the miscut direction and faceting. APL Mater. 7, 022515 (2019).

10. Pearton, S. J.; Yang, J.; Cary, P. H.; Ren, F.; Kim, J.; Tadjer, M. J.; Mastro, M. A., A review of Ga2O3 materials, processing, and devices. Appl. Phys. Rev. 5, 011301 (2018).

11. Åhman, J.; Svensson, G.; Albertsson, J., A reinvestigation of $\beta$-Gallium oxide. Acta Crystallogr. Sect. C: Cryst. Struct. Commun. 52, 1336-1338 (1996).

12. Li, M.-Y.; Shi, Y.; Cheng, C.-C.; Lu, L.-S.; Lin, Y.-C.; Tang, H.-L.; Tsai, M.-L.; Chu, C.-W.; Wei, K.-H.; He, J.-H., Epitaxial growth of a monolayer WSe2-MoS2 lateral pn junction with an atomically sharp interface. Science 349, 524-528 (2015).

13. Hsu, W.-T.; Zhao, Z.-A.; Li, L.-J.; Chen, C.-H.; Chiu, M.-H.; Chang, P.-S.; Chou, Y.C.; Chang, W.-H., Second harmonic generation from artificially stacked transition metal dichalcogenide twisted bilayers. ACS Nano 8, 2951-2958 (2014).

14. Kumar, N.; Najmaei, S.; Cui, Q.; Ceballos, F.; Ajayan, P. M.; Lou, J.; Zhao, H., Second harmonic microscopy of monolayer MoS2. Phys. Rev. B 87, 161403 (2013).

15. van der Zande, A. M.; Huang, P. Y.; Chenet, D. A.; Berkelbach, T. C.; You, Y.; Lee, G.-H.; Heinz, T. F.; Reichman, D. R.; Muller, D. A.; Hone, J. C., Grains and grain boundaries in highly crystalline monolayer molybdenum disulphide. Nat. Mater. 12, 554-561 (2013).

16. Zhang, X.; Zhang, F.; Wang, Y.; Schulman, D. S.; Zhang, T.; Bansal, A.; Alem, N.; Das, S.; Crespi, V. H.; Terrones, M.; Redwing, J. M., Defect-Controlled Nucleation and Orientation of WSe2 on hBN: A Route to Single-Crystal Epitaxial Monolayers. ACS Nano 13, 3341-3352 (2019).

17. Radisavljevic, B.; Radenovic, A.; Brivio, J.; Giacometti, i. V.; Kis, A., Single-layer MoS2 transistors. Nat. Nanotechnol. 6, 147-150 (2011).

18. Wang, Y.; Kim, J. C.; Wu, R. J.; Martinez, J.; Song, X.; Yang, J.; Zhao, F.; Mkhoyan, A.; Jeong, H. Y.; Chhowalla, M., Van der Waals contacts between three-dimensional metals and two-dimensional semiconductors. Nature 568, 70-74 (2019).

19. Amani, M.; Lien, D.-H.; Kiriya, D.; Xiao, J.; Azcatl, A.; Noh, J.; Madhvapathy, S. R.; Addou, R.; Santosh, K.; Dubey, M., Near-unity photoluminescence quantum yield in MoS2. Science 350, 1065-1068 (2015).

20. Lien, D.-H.; Uddin, S. Z.; Yeh, M.; Amani, M.; Kim, H.; Ager, J. W.; Yablonovitch, E.; Javey, A., Electrical suppression of all nonradiative recombination pathways in monolayer semiconductors. Science 364, 468-471 (2019).

21. Tongay, S.; Suh, J.; Ataca, C.; Fan, W.; Luce, A.; Kang, J. S.; Liu, J.; Ko, C.; Raghunathanan, R.; Zhou, J., Defects activated photoluminescence in two-dimensional semiconductors: interplay between bound, charged, and free excitons. Sci. Rep. 3, 2657 (2013).

22. Kresse, G.; Furthmüller, J., Efficiency of ab-initio total energy calculations for metals and semiconductors using a plane-wave basis set. Comput. Mater. Sci. 6, 15-50 (1996).

23. Wimmer, E.; Christensen, M.; Eyert, V.; Wolf, W.; Reith, D.; Rozanska, X.; Freeman, C.; Saxe, P. J. J. o. t. K. C. S., Computational materials engineering: Recent applications of VASP in the MedeA® software environment. 53, 263-272 (2016).

24. Perdew, J. P.; Burke, K.; Ernzerhof, M., Generalized gradient approximation made simple. Phys. Rev. Lett. 77, 3865 (1996). 
25. Klimeš, J.; Bowler, D. R.; Michaelides, A., Van der Waals density functionals applied to solids. Phys. Rev. B 83, 195131 (2011).

a

(I) $\mathrm{B}_{-} \mathrm{Ga}_{2} \mathrm{O}_{3}$

(II) Directed $\mathrm{MoS}_{2}$ (III) Aligned $\mathrm{MoS}_{2}$
nanoribbons

(IV) Transfer
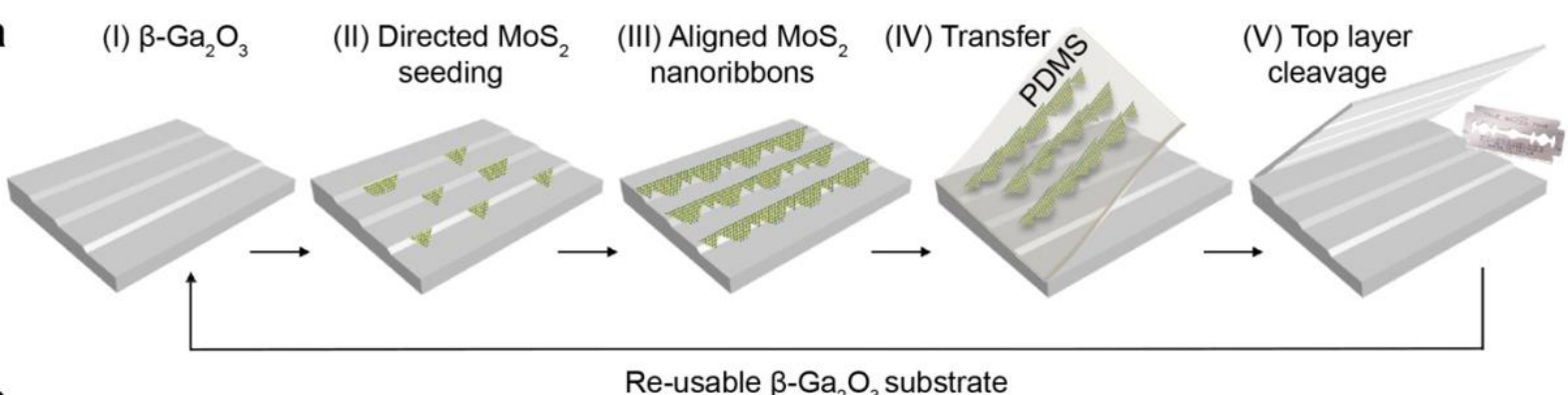

b

Re-usable $\beta-\mathrm{Ga}_{2} \mathrm{O}_{3}$ substrate
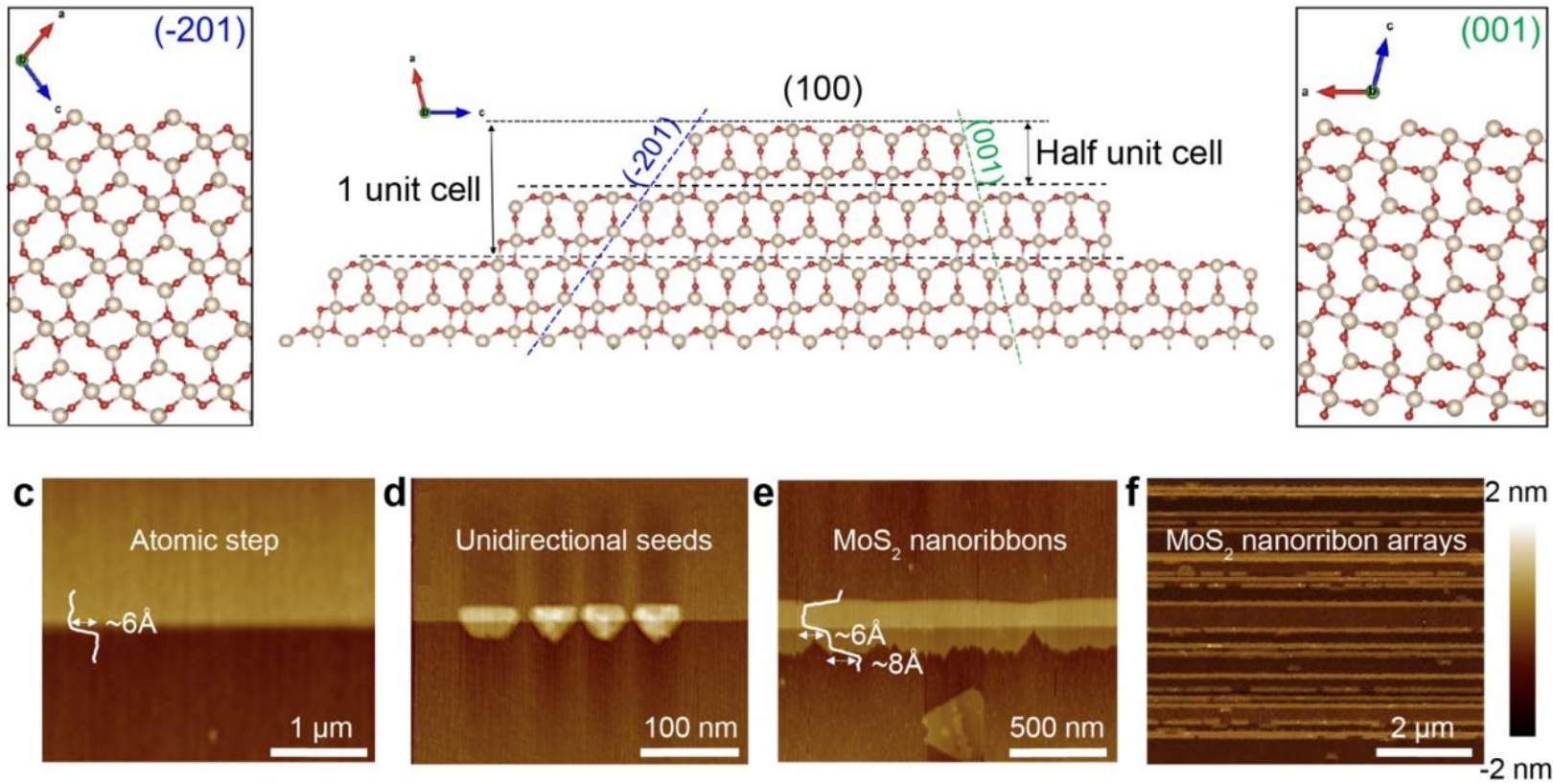

g

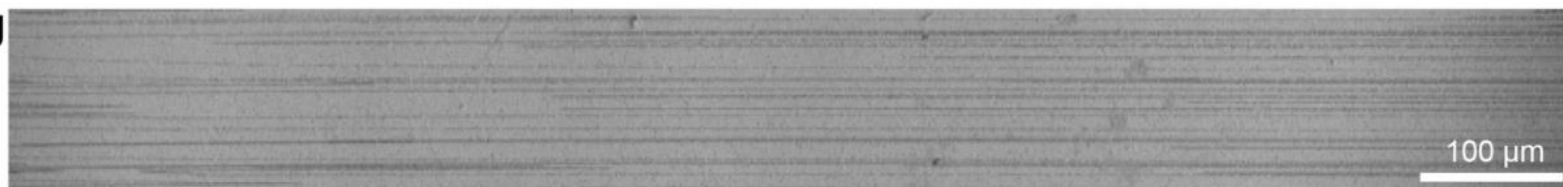

Fig. 1 | LDE growth of wafer-scale, globally aligned monolayer MoS2 nanoribbons. a, Schematic illustration depicts the sequential growth of the monolayer $\mathrm{MoS}_{2}$ nanoribbons along the intrinsically aligned ledges on $\beta-\mathrm{Ga}_{2} \mathrm{O}_{3}$ (100) substrate, which can be reused after a facile mechanical exfoliation. b, Computer generated crystal 
structures provide a cross-sectional view of both (-201) and (001) ledges on the (100) $\beta$ $\mathrm{Ga}_{2} \mathrm{O}_{3}$. c, Height profile along the well-defined atomic step on pristine $\beta-\mathrm{Ga}_{2} \mathrm{O}_{3}$ substrate helps determine the step height of $\sim 6 \AA$. $\mathbf{d}$, Nucleation of unidirectional MoS2 seeds along the ledge. e, A continuous $\mathrm{MoS}_{2}$ nanoribbon with asymmetric edges. $\mathbf{f}$, Dense arrays of aligned $\mathrm{MoS}_{2}$ nanoribbons. g, SEM image showcases the continuous growth of globally aligned $\mathrm{MoS}_{2}$ nanoribbons beyond millimeter-scale.
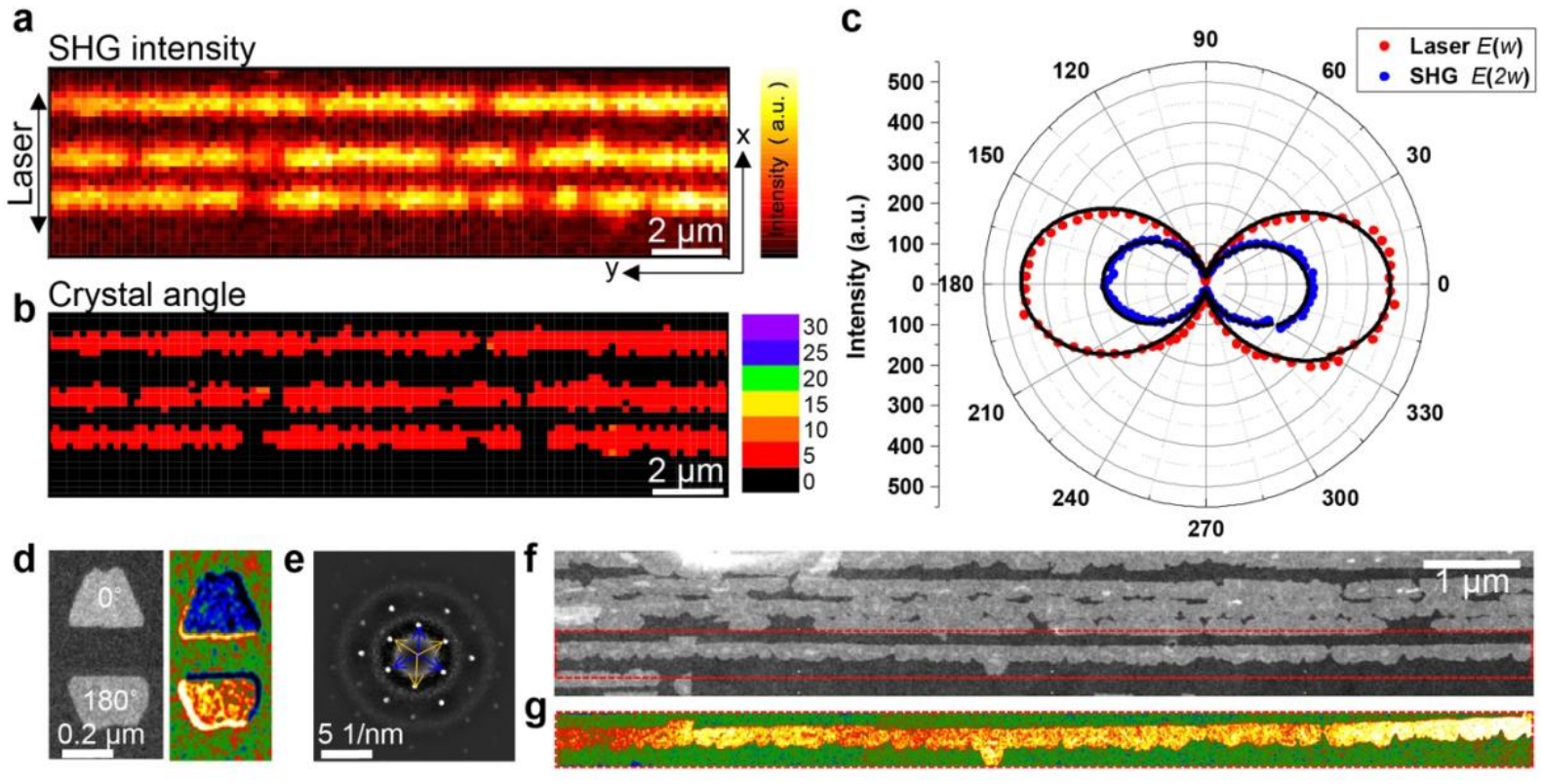

Fig. 2 | Polarization-resolved SHG measurement and DF-STEM characterization. a, The intensity map of the parallel component of SHG. The black arrow indicates the direction of incident laser polarization. $\mathbf{b}$, Map of the angle $(\boldsymbol{\theta})$ between the direction of laser polarization and the armchair direction of the $\mathrm{MoS}_{2}$ nanoribbons. c, Polar plot of the polarization-resolved SHG intensity and the backscattered laser light as a function of detection angles. d, (left) $\mathrm{MoS}_{2}$ mirror domains of $0^{\circ}$ and $180^{\circ}$ orientations can be clearly juxtaposed with the differential diffraction filtered STEM mapping of domain orientations (right). e, Corresponding convergent beam diffraction patterns of the oppositely oriented domains in (d). f, ADF-STEM image together with (g) differential diffraction filtered STEM mapping of the $\mathrm{MoS}_{2}$ nanoribbon highlighted in the red dotted rectangle collectively attests to the absence of grain boundaries which in turn confirms the single crystallinity of the LDE MoS2 nanoribbons. 

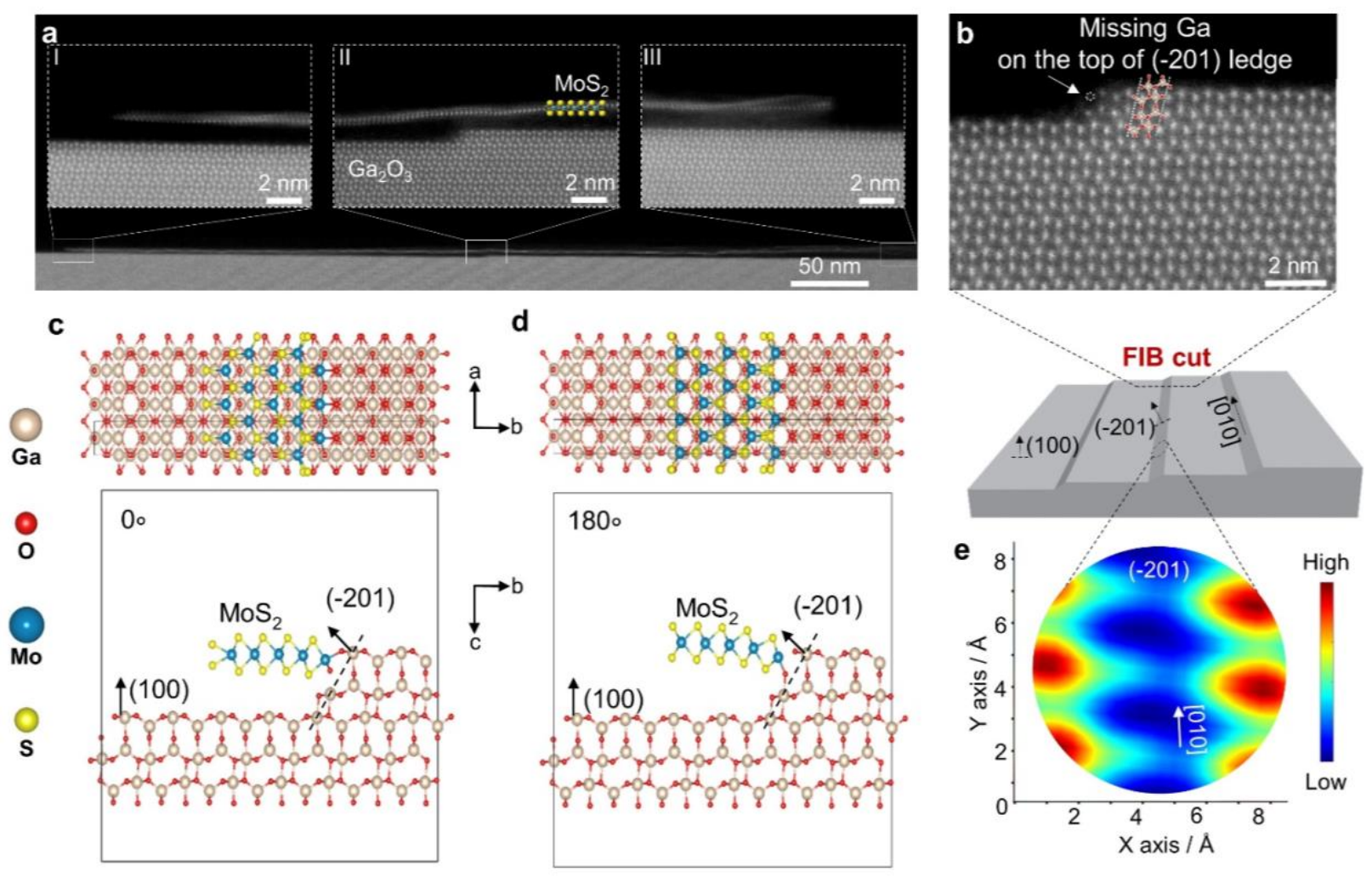

Fig. 3 | Atomically resolved imaging and proposed mechanism for LDE growth of

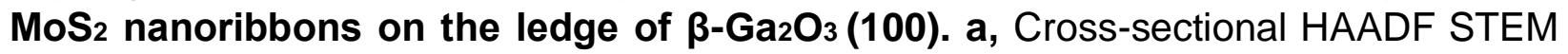
image of a $\mathrm{MoS}_{2}$ nanoribbon grown on the $\beta-\mathrm{Ga}_{2} \mathrm{O}_{3}(100)$ substrate. The insets provide atomically resolved views of different sections of the MoS2 nanoribbons on: (I) bottom terrace (left); (II) ledge (center); and (III) top terrace (right), respectively. b, Crosssectional HAADF HR-STEM image of $\beta-\mathrm{Ga}_{2} \mathrm{O}_{3}(100)$ substrate taken normal to the [010] direction reveals a missing $\mathrm{Ga}$ atom from the ledge. Computer-generated atomic models suggest two possible nucleation events on (-201) ledges with orientations toward (c) $0^{\circ}$, and (d) $180^{\circ}$, respectively. The presence of the ledge provides an energetically favorable docking site that breaks the energetic degeneracy by $\sim 2 \mathrm{eV}\left(0^{\circ}\right)$, thus unidirectionally orienting the seeding flakes. e, Potential energy surface (PES) mapping derived from the DFT calculations sheds light on the diffusion limited pathway to direct the growth of aligned $\mathrm{MoS}_{2}$ flakes into single-crystal nanoribbons. 
a

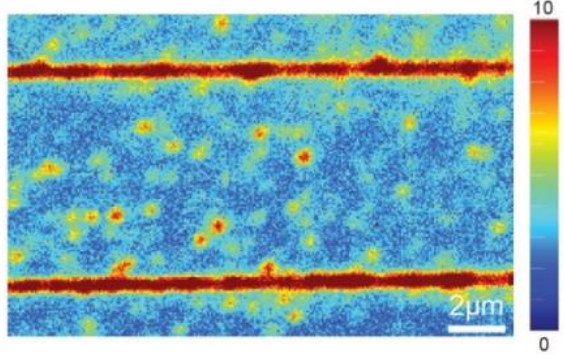

c

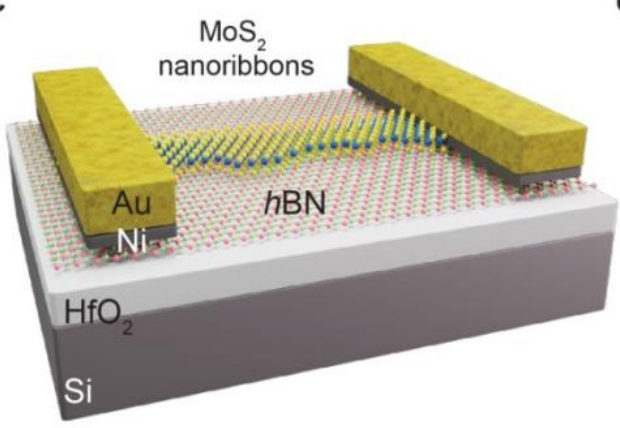

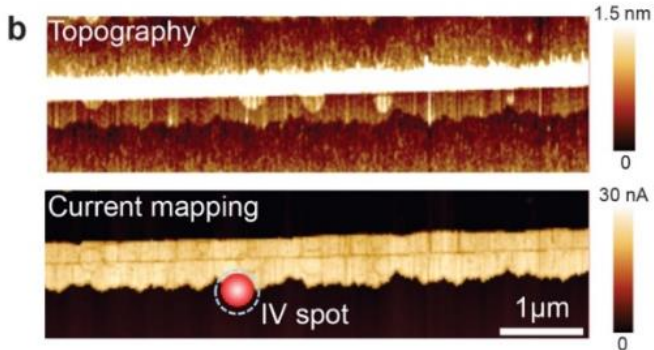

d

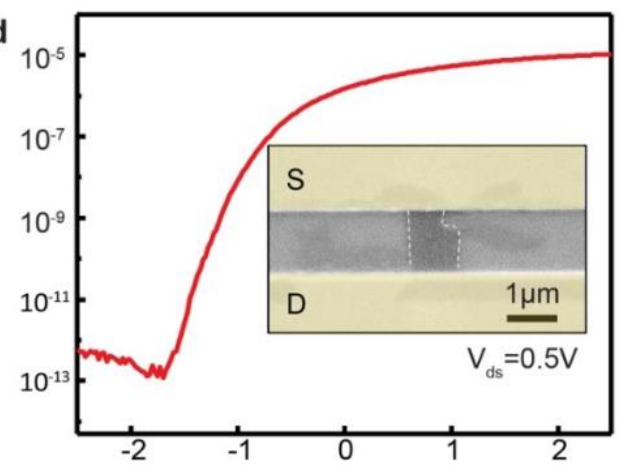

Fig. 4 | Optical, electrical characterizations and batch fabrication of $\mathrm{MoS}_{2}$ nanoribbon field-effect transistor. a, Hyper-spectral PL mapping displays a uniform wavelength distribution along the two parallelly aligned $\mathrm{MoS}_{2}$ nanoribbons. b, Topological (top) and the corresponding current mapping images (bottom) show the spatially uniform current profile of a MoS 2 nanoribbon at a constant tip voltage. c, Schematic illustration shows the device architecture comprised of a monolayer MoS2 nanoribbon stacked on top of a single crystalline $h \mathrm{BN}$ interface layer. d, Transfer characteristic of the MoS 2 nanoribbon/hBN field-effect transistor (FET, Inset shows the SEM of a MoS2 nanoribbon sandwiched between source and drain electrodes); the length and width of the device are $1 \mu \mathrm{m}$ and $0.39 \mu \mathrm{m}$, respectively, giving rise to an averaged electron mobility $\mu=65 \mathrm{~cm}_{2} \mathrm{~V}_{-1 \mathrm{~S}}-1$ at a drain voltage $\mathrm{V}_{\mathrm{ds}}$ of $0.5 \mathrm{~V}$. 